\title{
Nanoparticles and cancer therapy: A concise review with emphasis on dendrimers
}

\author{
Dhruba J Bharali \\ Marianne Khalil \\ Mujgan Gurbuz \\ Tessa M Simone \\ Shaker A Mousa \\ Pharmaceutical Research Institute \\ at Albany College of Pharmacy, \\ Rensselaer, NY, USA
}

Correspondence: Shaker A Mousa Professor of Pharmacology, Executive VP, Chairman of PRI, I Discovery Drive, Rensselaer, NY 12144, USA

Tel + I 5 I8 6947397

Fax +I 5186947576

Emailmousas@acp.edu

\begin{abstract}
The emergence of nanotechnology has had a profound effect on many areas of healthcare and scientific research. Having grown exponentially, the focus of nanotechnology has been on engineering diversified novel applications that even go beyond therapeutic activity; nanotechnology also offers the ability to detect diseases, such as cancer, much earlier than ever imaginable. Often, patients diagnosed with breast, lung, colon, prostate, and ovarian cancer have hidden or overt metastatic colonies. With the advent of diagnostic nanotechnology, these numbers are expected to greatly diminish. This review provides a brief description of nanoparticle (liposome, quantum dot, and dendrimer)-mediated cancer therapy in the last decade with an emphasis on the development and use of dendrimers in cancer therapeutics.
\end{abstract}

Keywords: nanoparticles, dendrimer, quantum dots, liposome

\section{Introduction}

Nanoscience is an emerging field that deals with interactions between molecules, cells and engineered substances such as molecular fragments, atoms and molecules. In terms of size constraints, the National Nanotechnology Initiative (NNI) defines nanotechnology in dimensions of roughly 1 to 100 nanometers (nm), ${ }^{1}$ but in boarder range it can be extended up to $1000 \mathrm{~nm}$. Particles that fall within this range appear to be optimal for achieving a number of important tasks as nano-carriers, including the alteration of a drug's reactivity, strength, electrical properties, and ultimately, its behavior in vivo. There is great interest in developing new nanodelivery systems for drugs that are already on the market, especially cancer therapeutics. Ideally, nanodelivery systems will allow for more specific targeting of the drug, thereby improving efficacy and minimizing side effects. By using nanotechnology in drug design and delivery, researchers are trying to push nanomedicine to be able to deliver the drug to the targeted tissue, release the drug at a controlled rate, be a biodegradable drug delivery system, and to be able to escape from degradation processes of the body.

Cancer is a disease that affects millions of Americans in all age groups and both sexes. According to the American Cancer Society, the probability of developing cancer during one's lifetime is one in two for men and one in three women. ${ }^{2}$ Most of the current chemotherapeutic agents on the market are low molecular weight agents with high pharmacokinetic volume of distribution both of which contribute to their cytotoxicity. Moreover, the low molecular weight of these chemicals makes them easily excreted, hence a higher concentration is ultimately required, and consequently a higher toxicity is unavoidable. Their low therapeutic index does not contribute favorably to this dilemma, as the needed concentration for the effective treatment must always be reached, but unfortunately the therapeutic levels are often exceeded. Additionally, these drugs when administrated alone, lack specificity and cause significant damage to noncancerous tissues. This results in serious, unwanted side effects such as bone marrow suppression, hair loss (alopecia), and the sloughing of the gut epithelial cells. ${ }^{3}$ 
Most chemotherapeutic agents have poor solubility and low bioavailability, and are formulated with toxic solvents. ${ }^{4}$ Thus, the use of nanocarriers allow for the preparation of low water soluble cancer medications in solid or liquid formulations. Doxil and Abraxane are the two well known US Food and Drug Administration (FDA)-approved nanoformulations currently available on market for cancer treatment. Doxil ${ }^{5-7}$ is a long circulating liposomal formulation of doxorubicin, which has shown significant improvements over its counterpart, free doxorubicin. The other FDA-approved nanomedicine, Abraxane ${ }^{\circledR}$ (Abraxis Bioscience, Los Angeles, CA), a treatment for metastatic breast cancer, is an albuminbound nanoparticle formulation of paclitaxel in the field of nanotechnology mediated cancer therapy.$^{8-10}$ Abraxane ${ }^{\circledR}$ for injectable suspension evades the hypersensitivity reaction associated with Cremophor EL, the traditionally used solvent for paclitaxel. This nanoparticle has a size around $100 \mathrm{~nm}$ and offers the ability to convert insoluble or poorly soluble drugs, avoiding the need for toxic organic solvents.

Having sprouted from a single concept some thirty years ago, nanotechnology has given rise to nanomedicine, among other applications including those associated with physics, biochemistry, and biotechnology, for creating molecular devices able to facilitate therapeutic and diagnostic procedures on the nanoscale. Thus, applications of nanotechnology have generated immense interest over past decade in various fields for diverse applications. This review will discuss the nanotechnological research that has been conducted regarding liposomes, quantum dots (QDs), and especially dendrimers in anticancer therapeutics over the past decade.

\section{Liposomes}

Liposomes are small lipid vesicles within the range of 50 to $1000 \mathrm{~nm} .{ }^{11,12}$ Because of their unique advantages over traditional drug therapy, including their ability to protect the drugs from degradation, targeting the drug to the site of action and reducing the toxicity and side effects of such drugs, liposomes have been studied extensively as drug carriers particularly for cancer therapy. ${ }^{13}$ Depending upon their size and number of bilayers, liposomes can be classified into three categories: multi-lamellar vesicles (MLV); large uni-lamellar vesicles (LUV); and small uni-lamellar vesicles (SUV). Liposomes can be classified in terms of composition and mechanism of intracellular delivery into five types: conventional liposomes (CL), pH-sensitive liposomes, cationic liposomes, immunoliposomes, and long-circulating liposomes (LCL). The major problems associated with liposomes are their stability, poor batch to batch reproducibility, difficulty in sterilization, and low drug loading capacity. Although liposomes have been extensively studied for last few decades, the only efficient nanoformulation available in the market is Doxil ${ }^{\circledR}$ (ALZA, Moutain View, CA).

\section{Quantum dots}

Semiconductor QDs are rapidly emerging as popular luminescence probes for many biological and biomedical applications owing to their extremely small size (approximately $10 \mathrm{~nm}$ in diameter), high photostability, tunable optical properties, and multimodality. ${ }^{14-18}$ Such inorganic-organic composite nanomaterials have shown extreme efficiency in cancer diagnosis in vivo, with their small size which facilitates unimpeded systemic circulation and attached targeting molecules, allowing for specific 'honing in' at neoplastic sites. ${ }^{19-22}$ Similar to other nanoparticles, QDs can be modified via conjugation of various surface molecules for targeted delivery. ${ }^{23,24}$ QDs also provide sufficient surface area to attach therapeutic agents for simultaneous drug delivery and in vivo imaging 25 as well as for tissue engineering. ${ }^{26}$ In vivo cancer targeting and imaging in living animals by QDs was first demonstrated by Gao and colleagues, ${ }^{19}$ where both subcutaneous injection of QD-tagged prostate cancer cells and systemic injection of multifunctional QD probes were used to achieve sensitive and multicolor fluorescence imaging of cancer cells. In a recent a study, Bagalkot and colleagues ${ }^{27}$ used QD-apatamer (Apt)doxorubicin (Dox) conjugate for targeted cancer therapy, imaging, and sensing. It was shown that this multifunctional nanoparticle system can deliver doxorubicin to the targeted prostate cancer cells and sense the delivery of doxorubicin by activating the fluorescence of QD, while allowing for simultaneous imaging of the cancer cells.

Though most of the major challenges associated with QD to make less toxic optical probes for biological applications have been overcome using different coating materials, under some conditions QDs can become cytotoxic. ${ }^{28}$ It was discovered that $\mathrm{CdSe}$ particles may leak cytotoxic cadmium ions after long-term exposure to ultraviolet (UV) light, while CdTe particles produce reactive oxygen species due to the loss of their protective coating after long-term circulation. In both cases, cytotoxicity and cell death were recorded. ${ }^{29-32}$ Research is ongoing to find suitable biocompatible and stable QD coatings. Nevertheless, QDs also found its application in the near infrared (NIR) imaging $(700-1000 \mathrm{~nm}){ }^{33}$ The use of NIR-QDs can maximize the depth of tissue penetration, allowing for more accurate and sensitive detection of photons in vivo, which is limited by absorption and light scattering in conventional imaging, allowing for more accurate and 
sensitive detection of photons in vivo. NIR-QD also may be a superior alternative because they can circumvent the problem of auto-fluorescence which is often associated with optical imaging of naturally-occurring compounds in animal tissue. Thus, these QDs have tremendous potential for in vivo imaging, and have already been used in various in vivo studies, including lymphatic mapping in animal models. ${ }^{34,35}$

\section{Dendrimers}

Dendrimers are a unique class of repeatedly branched polymeric macromolecules with numerous arms extending from a center, resulting in a nearly-perfect three-dimensional geometric pattern. Dendrimers can be synthesized via two major strategies: a) divergent methods and b) convergent methods, which differ in their direction of synthesis; either outward from the core or inwardly toward the core, respectively. Divergent methods were first introduced by Tomalia ${ }^{36}$ in the 1980's, where his group synthesized three-dimensional polyamindoamine (PAMAM) dendrimers by the growth of branches extending radially from a core site to the periphery. ${ }^{37}$ Furthermore, PAMAM dendrimers contain tertiary amines and amide linkages which allow for the binding of numerous targeting and guest molecules.

Hawker and Frechet are well known for having established the convergent strategies for the synthesis of dendrimers. ${ }^{38} \mathrm{In}$ convergent methods, dendrimer surfaces are first synthesized by gradually linking surface unit monomers together. When the growing surface wedges are large enough, several are attached to a suitable core to give a complete dendrimer. Polypropylenemine (PPI) and polyaryl ethers dendrimers are synthesized by using this convergent method.

Dendrimers have three components: an initiator core, branches, and terminal functional groups. The initiator core is in the heart of the molecule, and branches extend outward from it. The monomers attached to the core (G0), are called first generation monomers (G1) and two second generation monomers (G2) are attached to the each first generation monomers. Successive generations will form in this same manner, being two monomers attached to the monomer from the previous generation (Figure 1). The molecular weight of the dendrimer nearly doubles with each additional generation. ${ }^{39}$ Furthermore, terminal groups can be modified to obtain both a charged, and hydrophilic or lipophilic function for the desired biological and drug delivery application. ${ }^{40}$

The advantage of dendrimers is that they can be synthesized and designed for specific applications. They are ideal drug delivery systems due to their feasible topology, functionality and dimensions; and also, their size is very close to various important biological polymers and assemblies such as DNA and proteins which are physiologically ideal. ${ }^{41}$

Many of the properties of dendrimers include: ${ }^{42}$

1. Nanoscale sizes that have similar dimensions to important bio-building blocks, eg, proteins, DNA.

2. Numbers of terminal surface groups $(Z)$ suitable for bioconjugation of drugs, signaling groups, targeting moieties or biocompatibility groups.

3. Surfaces that may be designed with functional groups to augment or resist trans-cellular, epithelial or vascular biopermeability.

4. An interior void space may be used to encapsulate small molecule drugs, metals, or imaging moieties. Encapsulating in that void space reduces the drug toxicity and facilitates controlled release.

5. Positive biocompatibility patterns that are associated with lower generation anionic or neutral polar terminal surface groups as compared to higher generation neutral apolar and cationic surface groups.

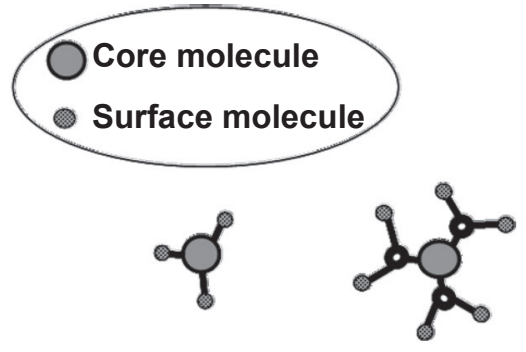

Generation: $\mathbf{G 0}$ Diameter: $1.4 \mathrm{~nm}$
G1 $1.9 \mathrm{~nm}$

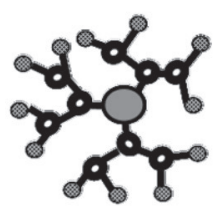

G2

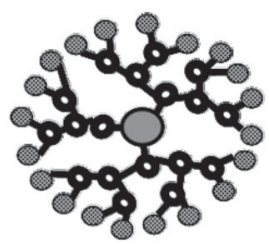

G3 $3.6 \mathrm{~nm}$

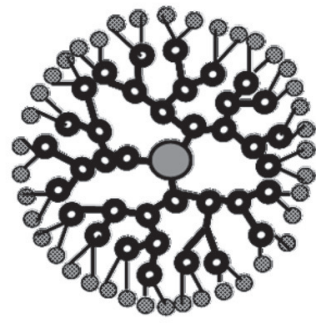

G4

$4.4 \mathrm{~nm}$

Figure I Graphical representation of dendrimers from core to generation $G=4$, showing the linear increase in diameter. 
6. Non- or low-immunogenicity associated with most dendrimer surfaces modified with small functional groups or polyethylene glycol (PEG).

7. Surface groups that can be modified to optimize biodistribution; receptor mediated targeting, therapy dosage or controlled release of drug from the interior space.

8. Ability to arrange excretion mode from body, as a function of nanoscale diameter.

\section{Dendrimer and anticancer drug delivery}

One of the major applications of dendrimers is as a delivery vehicle for various anticancer drugs. The structure and tunable surface functionality of dendrimers allows for the encapsulation/conjugation of multiple entities, either in the core or on the surface, rendering them ideal carriers for various anticancer drugs. There are numerous examples of dendrimer mediated targeted drug delivery.

In 2002, Jesus and colleagues ${ }^{43}$ had explored the possibility of a 2, 2-bis (hydroxymethyl) propanoic acid based dendritic scaffold as a delivery carrier for doxorubicin in vitro and in vivo. This dendritic nanoformulation, which contains doxorubicin covalently bound through a hydrazone linkage to a high molecular weight 3-arm polyethylene oxide; exhibits reduced cytotoxicity in vitro. However, in vivo biodistribution experiments showed minimal accumulation of the DOXdendrimer conjugate in vital organs, including the liver and heart, and increased half-life of doxorubicin compared to the free drug. Thus, it was hypothesized that proper choices of nanocarrier systems can increase the circulation half-life to effectively exploit the enhanced permeation retention (EPR) effect phenomenon and thus have tremendous potential to increase the efficacy of the drug to a greater extent.

In an attempt to improve the efficacy of doxorubicin, Lai and colleagues ${ }^{44}$ utilize photochemical internalization (PCI) technology for site-specific delivery of membrane impermeable macromolecules from endocytic vesicles into the cytosol. PCI technology has been demonstrated to successfully enhance the cytotoxicity of cancerous tissue by destroying the cytoplasmic membrane and facilitating the release of macromolecules entrapped in cytoplasmic vesicles. PAMAM dendrimers were conjugated to doxorubicin through the amide (PAMAM-amide-DOX) or the hydrazone (PAMAMhyd-DOX) bonds. Two different PCI strategies referred to as 'light after', and 'light before', where exposure to light is either after or before treatment with doxorubicin respectively, were applied to evaluate the cytotoxic effects of these doxorubicin PAMAM conjugates against $\mathrm{Ca} 9-22$, gingival carcinoma, cells. It was observed that only the 'light after' PCI treatment significantly increased the nuclear accumulation of doxorubicin from the PAMAM-hyd-DOX conjugates and thus exhibited higher cytotoxicity probably due to the synergistic effects. Both PCI strategies failed to improve the cytotoxicity of PAMAM-amide-DOX conjugates.

Many investigators have also explored the feasibility of cisplatin incorporation in dendrimers. One of the early examples is PAMAM dendrimer generation 3.5 conjugated to cisplation through the sodium carboxylate surface giving a dendrimer-platinate (dendrimer-Pt; $20-25 \mathrm{wt} \%$ platinum), which resulted in a fairly water soluble nanoformulation with the ability to release cisplatin slowly in vitro..$^{45}$ It was observed that this formulation showed superior activity over cisplatin when injected i.p. into mice bearing B16F10 tumor cells, whereas the dendrimer-Pt and cisplatin were equi-active i.p. against i.p. L1210. Also, when administered i.v. to treat a palpable s.c. B16F10 melanoma, the dendrimer-Pt displayed additional antitumor activity whereas cisplatin was inactive.

Zhou and colleagues ${ }^{46}$ synthesized poly (amide-amine) based dendrimers with a cyclic core and four direction branches using the method referred to as 'time-sequenced propagation technique'. Using this technique, they successfully synthesized dendrimers from generation 0.5 to generation 5.5. Further, dendrimers were then reacted with 1-bromoacetyl-5fluorouracil to form dendrimer- $5 \mathrm{FU}$ conjugates. In vitro release kinetics in PBS revealed that the release of 5FU depends on the generation of the dendrimer used for the conjugation, and indicated that such a dendritic polymer could be a promising carrier for the controlled release of antitumor drugs.

PEGylated dendrimers are one of the sub-classes of dendrimers that attract numerous scientists due to its prolonged blood circulation time, lower level of toxicity and relatively lower accumulation in different organs. Also, in vivo experiments show a significantly higher accumulation in the tumor tissues due to the EPR effect. ${ }^{47}$ PEG-dendrimers are generally synthesized by the conjugation of PEG or polyethylene oxide (PEO) chains to a multifunctional dendritic chain. A 2006 study by Lee and colleagues ${ }^{48}$ showed the feasibility of polyester-based dendrimer-PEO-doxorubicin conjugate to substantially inhibit the progression of DOX-insensitive C-26 tumor subcutaneously implanted in BALB/c mice. This dendrimer-PEO-doxorubicin conjugate also showed the ability to eliminate the tumors at certain doses and was found to be more or less the same as compared to an equal amount of Doxil, which is currently available for liposome formulation of DOX.

Additionally, Bhadra and colleagues ${ }^{49}$ used PEGylated PAMAM dendrimers for the incorporation of 5FU. Thus, as 
anticipated, it was observed that this is formulation is suitable for prolonged delivery of anticancer drugs by in vitro and blood-level studies in albino rats, without producing any significant hematological disturbances. PEGylation contributed an additional advantage to the dendrimer formulation by reducing drug leakage and hemolytic toxicity. This, in turn, could improve drug-loading capacity and stabilize such systems in the body.

\section{Dendrimers for targeted delivery}

In the last few years there has been a growing interest by various scientists to use dendrimers for targeted delivery especially in cancer therapeutics. The multifunctional nature of the dendritic network makes it easier for researchers to incorporate both the drug and the targeting moieties simultaneously, and thus can be used for active targeting. Furthermore, passive targeting of tumors can also be achieved through PEGylation on the surface of dendrimers. It is well established that PEGylated nanoparticles accumulate in the tumor tissues due to the EPR effect. On the surface of a variety of cancer cells, folate receptors are overexpressed. ${ }^{50}$ Folate-modified dendrimers target these cells via ligandreceptor recognition. Folic acid targeted dendrimers which are covalently conjugated with methotrexate specifically kill receptor-expressing cells after the intracellular delivery of the drug through receptor-mediated endocyctosis. ${ }^{51}$ Quintana and colleagues $^{52}$ synthesized an ethylenediamine core PAMAM dendrimer of generation 5 which was covalently attached to folic acid, fluorescein, and methotrexate. This complex provided targeting, imaging and intracellular drug delivery capabilities with 100-fold decreased cytotoxicity over free methotrexate.

The unique architecture of dendrimers allows for multivalent attachment of imaging probes as well as targeting moieties. Thus, it can be also used as highly efficient diagnostic tool for cancer imaging. Gadolinium (Gd)based magnetic resonance imaging (MRI) contrast agents (CAs) can operate using an approximately 100-fold less concentration than iodine atoms required for computed tomography imaging. They can be targeted to a single site which improves the sensitivity of imaging. Furthermore, the size of the complex prevents leakage from the bloodstream into fluids in the body, which also improves sensitivity and image clarity. ${ }^{53}$

\section{Dendrimer and carbon nanotube}

The utilization of dendrimers as 'nanotemplates' for carbon nanotube formation in a controlled manner has been a growing area of research interest since Choi and his group introduced the idea of utilizing PAMAM dendrimers for the synthesis of catalytic nanoparticles for single-walled carbon nanotube (SWNT). Thus, using this dendritic platform, chemical vapor deposition (CVD) nanotubes with a narrow diameter distribution between 1 and $2 \mathrm{~nm}$ were obtained. ${ }^{54}$ Later, in another study by Amama and colleagues, the use of fourth-generation PAMAM dendrimers as a platform for synthesizing multi-walled carbon nanotubes (MWCNTs) with systematically varied diameter distributions and defect densities by microwave plasma-enhanced chemical vapor deposition was demonstrated. This technique involved the utilization $\mathrm{Fe} 2 \mathrm{O} 3$ nanoparticles prepared via an interdendritic templating mechanism involving $\mathrm{Fe} 3+$ ions and an amine-terminated dendrimer. The diameter distribution and quality of MWCNTs can be manipulated judiciously by varying the concentration of the catalyst solution and the calcination temperature..$^{55}$ Alternatively, Pan and colleagues used PAMAM dendrimers to coat single-walled carbon nanotubes (SWCNTs) to reduce cytotoxicity and enhance cellular uptake. It was observed that dendrimer coated SWCNTs were nontoxic as compared to uncoated SWCNTs. Also, the uptake of uncoated SWCNTs in MCF-7 metastatic breast cancer cells was found to be significantly less than the dendrimer coated nanotubes, due to increased dendrimer mediated endocytosis. ${ }^{56}$ Therefore, a homogeneous coating of a glycodendrimers on SWCNT known to decrease cytotocixity also shows the potential for nanotubes to be utilized as protein-binding sensors. ${ }^{57}$

\section{Nanoparticles and toxicity}

Though in recent years there has been a rapid expansion in nanoparticle and nanotechnology research in consumer products, there is limited information on the possible toxic health effects on humans and the environment to date. To assess the toxicity in a systematic manner, there is an urgent need to investigate the intracellular and in vivo fate of the nanoparticulate systems vis-à-vis in surface properties and morphology. In an early study by Dunford and colleagues ${ }^{58}$ in 1997, it was demonstrated that titanium dioxide/zinc oxide nanoparticles used in sunscreen can catalyze oxidative damage to DNA in vitro and in cultured human fibroblasts. In 2004, at a nanoscale materials and toxicity conference, Nanotox 2004, Vyvvan Howard revealed his initial findings that gold nanoparticles have the ability to move across the placenta from mother to fetus when injected into pregnant rats. ${ }^{59}$ There are a few studies that have assessed of toxicity of carbon nanotubes. Shvedova and colleagues ${ }^{60}$ hypothesized 
that the probable dermal toxicity and morphological changes seen were due to accelerated oxidative stress in the skin after having been exposed to the SWCNT. Furthermore, in a separate study, the same group demonstrated that the exposure to unrefined SWCNT may lead to increased pulmonary toxicity due to oxidative stress. ${ }^{61}$ Other toxicity studies of carbon nanotubes describe the of cause granulomas in rats and mice after acute exposure. ${ }^{62}$ Crystalline silver nanoparticle-related cytoxicity in lesioned skin, growing human fibroblasts, and keratinocytes was demonstrated by Lam and colleagues ${ }^{63}$ and Poon and Burd. ${ }^{64}$ These studies, among others, have begun to reveal the toxicity of nanoparticles and will pave the way for progression in this field of study.

\section{Conclusion}

The biomedical application of nanoparticles has experienced exponential growth in the past few years; however, current knowledge regarding the safety of nanocarriers is insufficient. As these new drug delivery systems are brought to clinical trial we will begin to be able to identify any negative side effects associated with these compounds. Preliminary and complementary animal studies should be carried out to identify the risks associated with nanoparticle use, with particular attention paid to the elimination processes. Furthermore, very little attention has been paid to the environmental effects and the potential effects on the health of those manufacturing these particles. It is reported that only $1 \%$ of the total expenditure (US $\$ 1.2$ billion) has been spent on occupational health and safety research of nanotechnology. ${ }^{65}$ Considering the countless potential applications of nanoparticles in the health sector, particularly in cancer research, there is an urgent need for the development of safety guidelines by the government. Among the nanoparticulate carriers, dendrimers have tremendous potential in the applications involving multifunctional nanoparticulate systems combining targeting, imaging, diagnostics and therapy. Thus, this multifunctional, unique nanoparticulate carrier has the potential to detect diseases, deliver medications, and monitor the ability to change the current scenario of cancer research and diagnosis in real time.

\section{Disclosure}

The authors report no conflicts of interest in this work.

\section{References}

1. National Nanotechnology Initiative. What is nanotechnology? [online] [Cited on April 25, 2008]. Available from: http://www.nano.gov/html/ facts/whatIsNano.html.

2. American Cancer Society. Cancer Facts and Figures 2007 [online]. [Cited Sept 2007]. Available from: http://www.cancer.org/downloads/ STT/CAFF2007PWSecured.pdf.
3. Luo Y, Prestwich GD. Cancer-targeted polymeric drugs. Curr Cancer Drug Targets. 2002;2:209-226.

4. Kwon GS. Polymeric micelles for delivery of poorly water-soluble compounds. Crit Rev Ther Drug Carrier Syst. 2003;20:357-403.

5. Martin FJ. Clinical pharmacology and antitumor efficacy of DOXIL (pegylated liposomal doxorubicin). In: Lasic DD, Papahadjopoulos D, editors. Medical Applications of Liposomes. New York: Elsevier Science BV; 1998. p. 635-688.

6. Nishiyama N, Kataoka K. Current state, achievements, and future prospects of polymeric micelles as nanocarriers for drug and gene delivery. Pharmacol Ther. 2006;112:630-648.

7. Park JW. 2002. Liposome-based drug delivery in breast cancer treatment, Breast Cancer Res. 2002;4:95-99.

8. Sparreboom A, Scripture CD, Trieu V, et al. Comparative preclinical and clinical pharmacokinetics of a cremophor-free, nanoparticle albumin-bound paclitaxel (ABI-007) and paclitaxel formulated in Cremophor (Taxol). Clin Cancer Res. 2005;11:4136-4143.

9. Gradishar WJ, Tjulandin S, Davidson N, et al. Phase III trial of nanoparticle albumin-bound paclitaxel compared with polyethylated castor oil-based paclitaxel in women with breast cancer. J Clin Oncol. 2005;23:7794-7803.

10. Moreno-Aspitia A, Perez EA. Nanoparticle albumin-bound paclitaxel (ABI-007): a newer taxane alternative in breast cancer. Future Oncol. 2005; 1:755-762.

11. Torchilin VP, Weissing V, editors. Liposomes: a practical approach. 2nd ed. New York: Oxford University Press; 2003.

12. Fahmy TM, Fong PM, Park J, Constable T, Saltzman WM. Nanosystems for simultaneous imaging and drug delivery to T cells. AAPS J. 2007;9:E171-E180.

13. Torchilin VP. Targeted pharmaceutical nanocarriers for cancer therapy and imaging. AAPS J. 2007;9:E128-E147.

14. Bruchez M Jr, Moronne M, Gin P, Weiss S, Alivisatos AP. Semiconductor nanocrystals as fluorescent biological labels. Science. 1998;281:2013-2016.

15. Warren CW, Chan SN. Quantum dot bioconjugates for ultrasensitive nonisotopic detection. Science. 1998;281:2016-2018.

16. Jaiswal JK, Mattoussi H, Mauro JM, Simon SM. Long-term multiple color imaging of live cells using quantum dot bioconjugates. Nat Biotechnol. 2003;21:32-33.

17. Akerman ME, Chan WC, Laakkonen P, Bhatia SN, Ruoslahti E. Nanocrystal targeting in vivo. Proc Natl Acad Sci U S A. 2002;99: 12617-12621.

18. Alivisatos P. The use of nanocrystals in biological detection. Nat Biotechnol. 2004;22:47-52.

19. Gao X, Cui Y, Levenson RM, Chung LW, Nie S. In vivo cancer targeting and imaging with semiconductor quantum dots. Nat Biotechnol. 2004;22:959-960.

20. Wu X, Liu H, Liu J, et al. Immunofluorescent labeling of cancer marker Her2 and other cellular targets with semiconductor quantum dots. Nature Biotechnol. 2003;21:32-33.

21. Michalet X, Pinaud FF, Bentolila LA, et al. Quantum dots for live cells, in vivo imaging, and diagnostics. Science. 2005;307:538-544.

22. Bharali DJ, Lucey DW, Jayakumar H, Pudavar HE, Prasad PN. Folate-receptor-mediated delivery of InP quantum dots for bioimaging using confocal and two-photon microscopy. J Am Chem Soc. 2005;127:11364-11371.

23. Alivisatos AP, Gu W, Larabell C. Quantum dots as cellular probes. Ann Rev Biomed Eng. 2005;7:55-76.

24. Hoshino A, Fujioka K, Oku T, et al. Quantum dots targeted to the assigned organelle in living cells. Microbiol Immunol. 2004; 48:985-994.

25. Howarth M, Takao K, Hayashi Y, Ting AY. Targeting quantum dots to surface proteins in living cells with biotin ligase. Proc Natl Acad Sci US A. 2005;102:7583-7588.

26. Goldberg M, Langer R, Jia X. Nanostructured materials for applications in drug delivery and tissue engineering. J Biomater Sci Polym Ed. 2007;18:241-268. 
27. Bagalkot V, Zhang L, Levy-Nissenbaum E, et al. Quantum dot-aptamer conjugates for synchronous cancer imaging, therapy, and sensing of drug delivery based on bi-fluorescence resonance energy transfer. Nano Lett. 2007;7:3065-3070.

28. Derfus AM, Chan WCW, Bhatia SN. Probing the cytotoxicity of semiconductor quantum dots. Nano Lett. 2004;4:11-18.

29. Cho SJ, Maysinger D, Jain M, Roder B, Hackbarth S, Winnik FM Long-term exposure to CdTe quantum dots causes functional impairments in live cells. Langmuir. 2007;23:1974-1980.

30. Green M, Howman E. Semiconductor quantum dots and free radical induced DNA nicking. Chem Commun (Camb). 2005;1:121-123.

31. Choi AO, Cho SJ, Desbarats J, Lovric J, Maysinger D. Quantum dotinduced cell death involves Fas upregulation and lipid peroxidation in human neuroblastoma cells. J Nanobiotechnology. 2007;5:1.

32. Lovric J, Cho SJ, Winnik FM, Maysinger D. Unmodified cadmium telluride quantum dots induce reactive oxygen species formation leading to multiple organelle damage and cell death. Chem Biol. 2005;12:1159-1161.

33. Frangioni JV. In vivo near-infrared fluorescence imaging. Curr Opin Chem Biol. 2003;7:626-634.

34. Parungo CP, Colson YL, Kim SW, et al. Sentinel lymph node mapping of the pleural space. Chest. 2005;127:1799-1804.

35. Parungo CP, Ohnishi S, Kim SW, et al. Intraoperative identification of esophageal sentinel lymph nodes with nearinfrared fluorescence imaging. J Thorac Cardiovasc Surg. 2005;129:844-850.

36. Tomalia DA, Dewald JR; The Dow Chemical Corporation (Midland, MI). Dense star polymers having core, core branches, terminal groups; United States patent US 4,507,466. 1985 March 26.

37. Tomalia DA, Baker H, Dewald J, et al. Anew class of polymer:starburstdendritic macromolecules. Polym J. 1985;17:117-132.

38. Hawker CJ, Fre'chet JMJ. Preparation of polymers with controlled molecular architecture. A new convergent approach to dendritic macromolecules, J Am Chem Soc. 1990;112:7638-7647.

39. Tomalia DA. Birth of a new macromolecular architecture:dendrimers as quantized building blocks for nanoscale synthetic polymer chemistry. Prog Polym Sci. 2005;30(3-4):294-324.

40. Bai S, Thomas C, Rawat A, Ahsan F. Recent progress in dendrimer-based nanocarriers. Crit Rev Ther Drug Carrier Syst. 2006;23:437-495.

41. Gillies ER, Frechet JM. Dendrimers and dendritic polymers in drug delivery. Drug Discov Today. 2005;10:35-43.

42. Tomalia DA, Reyna LA, Svenson S. Dendrimers as multi-purpose nanodevices for oncology drug delivery and diagnostic imaging. Biochem Soc Trans. 2007;35(Pt 1):61-67.

43. Padilla De Jesús OL, Ihre HR, Gagne L, Fréchet JM, Szoka FC Jr. Polyester dendritic systems for drug delivery applications: in vitro and in vivo evaluation. Bioconjug Chem. 2002;13:453-461.

44. Lai PS, Lou PJ, Peng CL, et al. Doxorubicin delivery by polyamidoamine dendrimer conjugation and photochemical internalization for cancer therapy. J Control Release. 2007;122:39-46.

45. Malik N, Evagorou EG, Duncan R. Dendrimer-platinate: a novel approach to cancer chemotherapy. Anticancer Drugs. 1999;10:767-776.

46. Zhuo RX, Du B, Lu ZR. In vitro release of 5-fluorouracil with cyclic core dendritic polymer. J Control Release. 1999;57:249-257.

47. Maeda H, Wu J, Sawa T, Matsumura Y, Hori K. Tumor vascular permeability and the EPR effect in macromolecular therapeutics: a review. J Control Release. 2000;65(1-2):271-284.

48. Lee CC, Gillies ER, Fox ME et al. A single dose of doxorubicinfunctionalized bow-tie dendrimer cures mice bearing C-26 colon carcinomas. Proc Natl Acad Sci U S A. 2006;103:16649-16654.
49. Bhadra D, Bhadra S, Jain S, Jain NK. A PEGylated dendritic nanoparticulate carrier of fluorouracil. Int J Pharm. 2003;257(1-2): 111-124.

50. Lu Y, Low PS. Folate-mediated delivery of macromolecular anticancer therapeutic agents. Adv Drug Deliv Rev. 2002;54:675-693.

51. Patri AK, Kukowska-Latallo JF, Baker JR Jr. Targeted drug delivery with dendrimers: comparison of the release kinetics of covalently conjugated drug ad non-covalent drug inclusion complex. Adv Drug Deliv Rev. 57:2203-2214.

52. Quintana A, Raczka E, Piehler L, et al. Design and function of a dendrimer-based therapeutic nanodevice targeted to tumor cells through the folate receptor. Pharm Res. 2002;19:1310-1316.

53. Kobayashi H, Brechbiel MW. Dendrimer-based macromolecular MRI contrast agents: characteristics and application. Mol Imaging. 2003;2:1-10.

54. Choi HC, Kim W, Wang D, Dai H. Delivery of catalytic metal species onto surfaces with dendrimer carriers for the synthesis of carbon nanotubes with narrow diameter distribution. J Phys Chem B. 2002;106:12361-12365.

55. Amama PB, Ogebule O, Maschmann MR, Sands TD, Fisher TS. Dendrimer-assisted low-temperature growth of carbon nanotubes by plasma-enhanced chemical vapor deposition. Chem Commun (Camb). 2006; (27):2899-2901.

56. Pan BF, Cui DX, Xu P, et al. Cellular uptake enhancement of polyamidoamine dendrimer modified single walled carbon nanotubes. J. Biomed Pharm Eng. 2007;1:13-16.

57. Wu P, Chen X, Hu N, et al. Biocompatible carbon nanotubes generated by functionalization with glycodendrimers. Angew Chem Int Ed Engl. 2008;47:5022-5025.

58. Dunford R, Salinaro A, Cai L, et al. Chemical oxidation and DNA damage catalysed by inorganic sunscreen ingredients. FEBS Lett. 1997;418(1-2):87-90.

59. Wootliff B. Bristish Scientist: Nanoparticles might move from mom to Fetus. Small Times. [Cited Jan 14, 2004]. Available from: http://www. smalltimes.com/articles/article_display.cfm?Section=ARCHI\&C= Bio\&ARTICLE_ID=269201\&p=109.

60. Shvedova AA, Castranova V, Kisin ER, et al. Exposure to carbon nanotube material: assessment of nanotube cytotoxicity using human keratinocyte cells. J Toxicol Environ Health A. 2003;66:1909-1926.

61. Shvedova AA, Kisin E, Murray AR. Exposure of human bronchial cells to carbon nanotubes caused oxidative stress and cytotoxicity. Proc Soc Free Rad Research Meeting, European Section: Taylor and Francis Group; 2004.

62. Lam CW, James JT, McCluskey R, Hunter RL. Pulmonary toxicity of single-wall carbon nanotubes in mice 7 and 90 days after intratracheal instillation. Toxicol Sci. 2004;77:126-134.

63. Lam PK, Chan ES, Ho WS, Liew CT. In vitro cytotoxicity testing of a nanocrystalline silver dressing (Acticoat) on cultured keratinocytes. Br J Biomed Sci. 2004;61:125-127.

64. Poon VK, Burd A. In vitro cytotoxity of silver: implication for clinical wound care. Burns. 2004;30:140-147.

65. Maynard AD. Nanotechnology: a research strategy for addressing risks. Woodrow Wilson International Center for Scholars: Project on emerging nanotechnologies. 2006. [Cited on Nov 10, 2008]. Available from http://www.nanotechproject.org/file_download/files/PEN3_Risk.pdf. 
